\title{
Dansei Konenki: Narratives of Male Menopause in Contemporary Japan
}

\author{
Tomoko Sakai, B.Sc. *
}

\begin{abstract}
Previous research has focused on cross-cultural comparisons of illnesses, such as depression and senile dementia, though few have studied the actual processes by which these illness categories become separated from their roots and adopted in a different locale; in anthropological terms, their "indigenization." Through anthropological fieldwork conducted from June to September of 2003, this paper explores how dansei konenki, or male menopause, has found a niche in contemporary Japan, as well as the defining features of the country that may explain this phenomenon. Based on this research, I argue that the indigenization of dansei konenki embodies a particular sociohistorical moment in Japan-namely, that of the long-running economic decline in recent years.
\end{abstract}

\section{INTRODUCTION}

Dansei konenki, a literal translation of the English term "male menopause," may be a term that is not familiar to many readers. The phrase might invoke an obscure, eccentric illness that only exists in exotic, nonwestern countries. On the contrary, male menopause-a paradoxical term in itself-is a strictly biomedical illness category, originating in the West (a). While it has never become well known in America, the country where it was codified, it has become a very well-known disease in Japan. Intriguingly, the term has not successfully laid its roots in its place of origin, but has somehow found its appeal in contemporary Japan.

In the summer of 2002, I conducted archival and ethnographic research on the scientific construction and lived experience of dansei konenki in Japan. During this period, I interviewed several clinicians who specialize in treating dansei konenki, as well as patients afflicted with the disease. In addition, I conducted participant observation at one clinic, which specializes in the treatment of this disorder, and in the homes and work environments of several patients-one of whom I document in this paper. Through the analyses of these data, I argue that the indigenization of dansei konenki

\footnotetext{
* To whom correspondence should be addressed: 1727 Waverley Street, Palo Alto, CA 94301.

E-mail address: daybyday03@yahoo.co.jp.
}

has been fueled by and is contingent upon a particular socio-historic moment in Japan. A movement embodied by an extended economic recession, and multitudinous shifts in family values, work ethics, and gender roles that the economic decline has induced in Japanese society over the past decade.

Interestingly, this illness category has become largely divorced from its roots and taken on a distinctly different set of meanings and definitions-both in the medical community and the larger society-that reverberate within the specific context of contemporary Japan. A process frequently referred to as the localization, or indigenization of an illness category in recent anthropological literature, it is manifested in multiple layers in Japanese society: first, the western, biomedical concept of dansei konenki is grafted onto local knowledge about health and the male body, and is subsequently transformed. For example, leading Japanese physicians involved in the treatment of this condition contend that a decrease in testosterone levels leads to an imbalance in the autonomic nervous system (b), triggering chronic fatigue, shortness of breath, and

(a) The first scientific paper discussing this disorder was published in the Journal of American Medical Association (JAMA). In its discussion, the paper notes that "the evidence enlisted in this study strongly suggests the possibility that men also suffer from menopausal syndromes typical of women, due to endocrine disruptions in climactic years." 
bad peripheral circulation; and that obesity and smoking can negatively affect the circulating levels of the hormone. In contrast to the overwhelming emphasis placed on male sexuality and its decline in the western discourse of male menopause (c), its indigenized counterpart in contemporary Japan embodies distinctly different theories of causation and physical manifestations.

The economic decline has proven to be a powerful force in reshaping important social values in the everyday lives of the Japanese-middle age men in particular-such as family values, work ethics, traditional corporate structures, and gender categories. As the illness narrative of Yoshiharu Sakaguchi, a patient diagnosed with dansei konenki will show, the drastic effects of the dragging recession on middle to old-age men-such as large-scale layoffs and major transformations in the traditional structure of companies-all signify an increasingly hostile and insecure environment for this particular population. Yoshiharu Sakaguchi is an executive of a multi-national trading company and the head of the Toyama branch. $\mathrm{He}$ was diagnosed with konenki while preparing for early retirement, after the company decided to close the Toyama (d) branch due to budget restraints. His narrative depicts the increasing difficulties that the creators of the current economic prosperity confront today-and accordingly, the diagnosis of konenki has added onto those distresses. For example, concurrent with the mild social stigma of being a "workaholic" who has neglected fatherly duties, which are becoming idealized as male virtues, the diagnosis also symbolizes a deprivation of masculinity for Yoshiharu Sakaguchi. Thus, dansei konenki in contemporary Japan has taken on meanings that reflect the multitude of changes and its effects that have resulted from this particular socioeconomic context.

\section{THEORETICAL RELEVANCE}

Much of medical anthropological literature has focused on instances where biomedical illness categories come in contact with non-western locales. While some works have focused on biomedical illness categories that face substantial resistance for public acceptance in non-western contexts, others document

(b) An unfamiliar concept in the West, jiritsushinkei shicchoushou (literally translated as 'autonomic imbalance,' is a popular term in the Japanese medical language. The condition is characterized by a broad set of physical and mental symptoms-such as headaches, insomnia, loss of appetite, vertigo, and mild depression-which all have unidentifiable or unspecific causes. Some physicians categorize the condition into three major groups: innate, psychological, and nervous-system based autonomic imbalance. (Masahiro Inode. Jiritsushinkei Shicchoushou. Takahashi shoten: 1994 the acceptance and subsequent indigenization of biomedical illnesses.

In No Aging in India: Alzheimer's, the Bad Family, and Other Modern Things (1), for example, Lawrence Cohen tackles the puzzling observation of how Alzheimer's in India is largely denied to exist. Simply put, in contrast to the U.S., where the pathologies of old age can be predominantly discussed as an unemotional and isolated medical issue, Cohen found the discourse of senility and aging in India to be intensely moral, emotional and cultural. These discrepancies, Cohen argues, may explain the apparent difficulty that Indians have in acknowledging Alzheimer's and senile dementia as a disorder that afflicts their own people, where the elderly are well-cared for and very much part of the traditional Indian joint family.

Conversely, the ethnography written by Paul Farmer looks at instances where biomedical illness categories with a western origin are taken up by non-western locales and are subsequently indigenized; similar to the case of male menopause in contemporary Japan. Paul Farmer's 1993 text, AIDS and Accusation: Haiti and the Geography of Blame documents how, as the AIDS pandemic spread in Haiti-most probably by the increased contacts between Haitian sex-workers and gay tourists in the early 1980 's - local meanings, as well as theories of causation and agency were grafted onto the biomedical definition of AIDS (2). In spite of efforts to educate the public about the scientific explanation and the prevention methods of AIDS, the majority of the destitute population attributed theories of voodoo curses, Haitian black magic, and white American racism to this illness in desperate attempts to assign blame and find a cure.

Like Farmer's work, the study of dansei konenki in contemporary Japan addresses the same issues of the adoption, transformation, and subsequent indigenization of an illness category with its roots in the West. However, the uniqueness of this case study lies in the fact that male menopause has become much more rapidly and readily acknowledged as a sound, biomedical illness in Japan, in contrast to the western countries where it was initially codified. In other words, this paper expands the analytic framework of Farmer by looking at the indigenization of a biomedical illness that has yet to be commonly recognized by the larger societies in the West.

(c) Although it is a topic of great interest, an elaborate discussion on the cross-cultural differences between the symptoms of male menopause from the Western and the Japanese perspective are avoided due to the limited scope of this research paper.

(d) Toyama is a small city 90 miles west of Tokyo, facing the Sea of Japan. 


\section{ETHNOGRAPHY OF ISHINKAI UROLOGY CLINIC}

The observation of clinical encounters at Ishinkai Urology Clinic exemplifies how dansei konenki, as a new illness category, is diagnosed, represented, and treated in ways which are clearly not straight imports from the western biomedical discourse of this disease, where its concept originated. For example, Dr. Hiromi Yokoyama, the director of the clinic, and leading expert on the diagnosis and treatment of dansei konenki, uses an original questionnaire as his primary diagnostic tool, which patients fill out during each visit, along with a thorough medical examination, including the measurement of circulating testosterone levels. It is divided into three sections: psychological/autonomic nervous symptoms, masculinity check, and symptoms of the urinary organs. Category 1 enlists criteria such as anxiety, irritability, fatigue, depressive mood, insomnia, and hot flashes; category 2 lists the frequency of sex and sexual desires, and category 3 asks about the frequency and uncomfortable symptoms accompanying urination. The patient ranks each diagnostic criterion from 0-3, indicating the degree of severity he is experiencing.

Additionally, in contrast to defining dansei konenki as simply a consequence of declining testosterone levels, as is customary in the United States, Japanese doctors have formulated their own diagnostic requirements for this new illness category: a conspicuous example of an illness category that is re-invented in the local environment. Dr.Yokoyama notes that the myriad of symptoms patients exhibit, such as hot flashes, headaches, chronic fatigue, and lack of sexual appetite, are caused by jiritsushinkei shicchoushou (autonomic imbalance). Additionally, they must follow a cycle of ebbs and flows: if any one symptom persists, then it is not caused by menopause.

As for testosterone levels, his theory holds that the large difference between circulating free testosterone levels before and after a man enters his middle age (e) is what characterizes male menopause. Hence, rather than setting an absolute standard of hormone levels to distinguish normal from abnormal ranges - one of the principal tenets of biomedicine - diagnostic standards are set differently for each individual. Thus, there are no strict diagnostic cutoffs for what is considered to be a physiologically normal or abnormal level of testosterone (f). Many times, Dr.Yokoyama will let the

(e) Here, I define "middle age" as the age range from the mid 40's to mid 50 's.

(f) The concept of applying a relative scale of individual hormone levels to diagnose a condition is not a common practice in Japan, and is characteristic of Dr.Yokoyama's own theory and treatment methods. Most medical institutions set a reference level, which is typically around $13-15 \mathrm{pg}$ free testosterone $/ \mathrm{ml}$ blood. patient decide on the dosage of testosterone administration, according to his subjective discretion.

Another physician pioneering the treatment of dansei konenki in Japan has his own set of standards for diagnosing the illness. A well-known cardiovascular specialist, Dr. Fuminobu Ishikura of Osaka University Medical School defines dansei konenki as a comprehensive term incorporating erectile dysfunction, cardiovascular abnormalities, and clinical depression (3). Interestingly, these two physicians come from two distinct backgrounds of medicine-Dr.Yokoyama from urology and prostate diseases, and Dr. Ishikura from the cardiovascular system. Thus, both doctors accommodate specific knowledge about their specialties to construct an illness category that fits their own definition of male menopause $(\mathrm{g})$.

Treatment methods have also undergone significant indigenization and appropriation, formulated to reverberate within local contexts. The most prominent example is Dr.Yokoyama's three main methods of treatment which he almost always combines for any patient - Testosterone replacement therapy (TRT), kanpo (h), and counseling - in contrast to the standard Western treatment which is solely confined to hormone injections. Only in cases of severe clinical depression, will he refer his patients to a psychiatrist. Dr. Yokoyama also uses supplements such as fukoidan (i), a type of dietary fiber extracted from seaweed, as part of his treatment methods. Dr. Ishikura, on the other hand, asks the first-time patient to complete a comprehensive questionnaire used in diagnosing clinical depression, and requires a session of relaxation methods, more commonly known as jiritushinkei kunren hou, or training of the autonomic nervous system, in addition to drug prescription and psychological counseling.

Other means of the local indigenization of dansei konenki are manifested in Dr.Yokoyama's numerous articles and publications. In one article entitled "Male

(g) Dr.Yokoyama and Dr.Ishikura are two prominent physicians involved in the treatment of male menopause. Their theories, as well as their clinic/hospital work, are completely independent from one another.

(h) Traditional Chinese herbal medicine. In her ethnography East Asian Medicine in Urban Japan (U.C.Press: Berkeley, 1980), Margaret Lock describes in detail how Western medicine and kanpo co-exist in dominant medical theories, as one popular treatment method for female menopausal symptoms is the use of these Chinese herbal medicine. The uniqueness of these remedies us the emphasis placed on individual differences - in other words, combining different herbs for different individuals, even if they exhibit similar symptoms.

(i) Although research on fukoidan is still in nascent stages, some studies have been done-including those by the National Cancer Institute, which has reported the apoptosis-inducing effects of fukoidan in cancerous cells. Dr.Yokoyama is, however, unique in his use of fukoidan to alleviate konenki symptoms. 
menopause, Female Menopause" he wrote for Anatani E-ru (An Eire to You) (4), a monthly subscription magazine targeted for reader audiences in their 50 's. Its pages are devoted to discussing certain kinds of food that are effective in alleviating male and/or female menopausal symptoms, as well as certain personalities and professions in which there is a high or low incidence of patients with konenki. The idea of food as medicine, an emphasis on harmony, balance, and equilibrium promoting an optimal state of being are characteristic of traditionally East Asian concepts of health. In terms of daily diet, Dr.Yokoyama encourages readers to eat "sticky/slimy" food, such as fermented soybeans, a relative of the taro root, and okra, all of which supposedly have properties that "balance hormone levels, repair prostate and other male functions." Beer is discouraged because one of its main ingredients, hop, contains estrogen-like compounds (4).

Personality-wise, men with "a strong sense of responsibility, a keen sense of competition who are punctual, impatient, and always hungry for success" will have a stronger tendency to develop menopausal symptoms compared to those who are "stable in their mental states, and maintain [their] own pace of life, unaffected by the environment." These translate into vulnerable professions - those that use the brain more than the body - such as corporate workers, company executives, and those who do a lot of deskwork. By contrast, gym teachers, military personnel, and construction site workers have a lower incidence of dansei konenki (4).

With initial research beginning in the U.S. in the 1940 's (5), the possibility that men may experience menopausal symptoms, and the use of the term as diagnostic, was first introduced to Japanese medical professionals at symposia held by prestigious academic institutions, such as the General Assembly of the Japan Medical Congress and the Japanese Urology Association. However, physicians involved in the treatment of konenki today claim that it received very little attention or understanding back then. Since then, the concept has gradually gained attention and acceptance from both the medical community as well as the general public - closely following the economic downfall which has resulted in socially significant events such as massive salary and job cuts among middle-age workers. By 2003, the institutions named above were seen to give much attention to the subject, and acknowledged the need for a collective effort to further new studies and reach an agreement about a standard definition, diagnosis, and treatment for this emerging illness category. Today, several university and public medical centers, such as St.Marianna Medical University and Tokyo Women's Medical University, have founded Departments of Andrology, holding outpatient hours specifically for dansei konenki.

According to one study, a random survey of 90 men between the ages 45 and 60 , using a questionnaire and interview, revealed that roughly $20-40 \%$ identified with the major symptoms associated with konenki (3). One of the pioneers in the diagnosis and treatment of dansei konenki, the Department of Urology at Kansai Medical University has also reported that it had diagnosed approximately 150 patients with the disorder-roughly 90 of whom are currently receiving testosterone replacement therapy-since it founded its outpatient clinic in January 2002 (2). Unfortunately however, large-scale, formal studies investigating the general incidence of dansei konenki, as well as the precise number of physicians or medical facilities which treat the condition in Japan today have yet to be conducted. The lack of an agreement on the specifics of the disorder, including its diagnosis and treatment by the Japanese medical community, lies at the root of the problem, and efforts to expedite the formulation of standard concepts regarding this novel illness category is much needed.

Participant-observations at Ishinkai Urology Clinic suggest that dansei konenki, as an indigenized illness category in contemporary Japan, has also taken on a socially significant function: the category serves to restore the social functionality of the patient, hence contributing to the recovery of the function of his family, his company, and the dire economic climate of the society. This stands in stark contrast to the general representation of male menopause in the U.S., where the discussion is heavily centered around issues of sexuality. Indeed, many American physicians contend that male menopause is simply another way of describing sexual/erectile dysfunction in middle-age men (3).

The rapid rise in the number of male patients who are visiting konenki outpatient clinics today, reflects the collective need for such an illness category in the particular socio-economic climate of contemporary Japan. The prolonged economic recession and decline starting from the early 1990's signified the demise of old corporate rules, such as shushin koyou, guaranteed lifetime employment, and nenko joretsu, a system closely following the ingrained ideas of East Asian filial piety, whereby one's position in the company directly reflects the years of service to the organization. Once the trademarks of Japanese corporations, shushin koyou and nenko joretsu both contributed to the illusion of a man's company seeming like his alternative family. The rapid demise of old corporate models have deprived men of their job security, which was once guaranteed, inducing notions of fear, instability, and anxiety as the 
burden of supporting his family continues to fall on his shoulders (6). The driving force behind the indigenization of dansei konenki, the need to give a voice to and contribute to the restoration of the man's social functionality, becomes understandable under this particular socio-economic context.

\section{YOSHIHARU SAKAGUCHI}

The following illness narrative of a 55 year old patient, illustrates how the indigenized illness category of dansei konenki has been fueled by, and is contingent upon a particular socio-economic moment in Japan, embodied by an extended economic recession and the paradigmatic shifts in family values, work ethics, and gender roles that it has induced over the past decade. Yoshiharu Sakaguchi had just received an order from the headquarters of his company to return to Tokyo after July, when the Toyama branch was scheduled to close due to radical budget cuts.

The long-term recession has also induced major structural changes that have marked a transition from the traditional to the new, "American" economic model. During the period of rapid economic growth in the 1970 's, admission to a prestigious university, through a competitive entrance exam, secured one's position on the elite track. In turn, a good university name guaranteed a job in a good company, which lead to lifetime employment, or shushin koyou (j). Under this system, one's company essentially became an alternative family, where individuals worked with a sense of security and interdependency with one another. After the downward spiraling of the economy in the early 1990's and subsequent demise of this corporate model, men were suddenly facing an age of insecurity, where lay-offs became common, and finding a new job was extraordinarily difficult for middle-age men. This especially held true for individuals who had administrative positions and lacked specific marketable skills and knowledge needed for re-employment (6).

Mr. Sakaguchi suffers from a typical set of symptoms said to accompany dansei konenki: a bad case of insomnia, night sweats and hot flashes during the day, mild depression, chronic fatigue, and prostate problems, and is currently receiving testosterone injections. Mr. Sakaguchi says he was primarily relieved when everything could be finally explained and treated, although the reaction contained inherent mixed emotions. Like many patients, the diagnosis of konenki fixes the idea of a new-found anxiety by signifying a rapid and irreversible degeneration of the masculine body to the patient.

(j) This is true mostly of men, since it was not typical for women to enter the work force after school until the 1990's.
Through the process of treating his condition, Mr. Sakaguchi and his wife both acknowledge the reestablishment of a mutual understanding and new-found appreciation for each other: an instance of a favorable role that konenki has played in his life. Thus, the reverberations of this indigenized illness in the life of Mr. Sakaguchi are pluralistic and exist in constant tension with one another-unlike many one-sided theories written on the effects of indigenized biomedical diseases. The diagnosis of dansei konenki seemed to have enhanced both the quantity and quality of communication between the Sakaguchis compared to the earlier years of their marriage, when Mr. Sakaguchi was much more reticent and stoic-minded.

Indeed, their experience points to another change, induced by the demise of the old economic paradigm, that the current konenki generation has to face and adopt. Posters of a young male pop star holding his new born son under the caption "We Don't Call Men Who Don't Participate in Child-Rearing Fathers," plastered throughout the subway stations around Tokyo, indicates the idealization of men as active participants of child rearing and household duties has as another prominent feature of the recent shifts in social values. Concurrent with this change, there seems to be a mild social stigma for men of his generation, for having been a "workaholic salary-man" who has neglected fatherly duties. Thus, men like Mr. Sakaguchi are confronting changes in the modern ideals of younger men: whereas male virtues traditionally embraced values such as stoicism, financial authority, and non-cooperation of household chores, modern young men are largely expected to be open and vocal in speaking their minds, viewing the opposite sex as equally competent players in the work force, and taking an active role in domestic activities.

\section{CONCLUSION}

Through the ethnographic account of a urology clinic and the illness narrative of one patient, I have tried to illustrate the process by which dansei konenki has become largely divorced from its Western roots, and has taken on distinct definitions that reverberate within the specific context of contemporary Japan. This indigenization, I have argued, has been fueled by and is contingent upon two key social contexts: the extended economic recession and the major shifts in traditional corporate structure, work ethics, family values, and gender roles that have resulted because of this social climate.

To be sure, male menopause is a relatively minor illness in terms of the degree to which it affects the physical and mental health of the patient and his or her family, as do AIDS or Alzheimer's disease. While 
illnesses that more ostensibly threaten the livelihood of patients have long been the focus of medical anthropologists writing about biomedical indigenization, a disorder like male menopause is interesting in its own right. In future studies, it would be of great interest and contribution to the field to further explore the indigenization of biomedical illness categories in traditionally non-western locales, and the socio-cultural forces that mediate and drive this process.

\section{REFERENCES}

1. Cohen, Lawrence. No Aging in India: Alzheimer's, the Bad Family, and Other Modern Things. 10-83; 1980.

2. Farmer, Paul. AIDS and Accusation: Haiti and the Geography of Blame. 1-27; 1993.

3. Ishikura et al. 47th Convention, Japanese College of Cardiology. Sept.13, 1999.

4. Yokoyama, Hiromi. Female Menopause, Male Menopause. Anatani E-ru: 22-26; Sept. 2002.

5. Heller, C.G., and Nyers, G.B. The Male Climactic: its symptomology, diagnosis, and treatment. JAMA 126: 472; 1944.

6. Onishi. Masami. The Dynamism of Japanese Human Resources Strategies. 7-132; 1997.

Tomoko Sakai received her B.S. degree in Biological Sciences from Stanford University in 2003, and is currently applying to medical schools. Her research interests include medical anthropology, bioethics, and international medicine. 